\title{
An efficient three component one-pot synthesis of some new octahydroquinazolinone derivatives and investigation of their antimicrobial activities
}

\author{
Niraj K. Ladani, Manish P. Patel, Ranjan G. Patel* \\ Department of Chemistry, S. P. University, Vallabh Vidyanagar-388 120, Gujarat, India \\ E-mail: patelranjanben@yahoo.com
}

\begin{abstract}
An efficient and convenient procedure has been developed for the synthesis of some new octahydroquinazolinones 5a-p in good yields. They have been achieved by the reaction between corresponding tetrazolo[1,5-a]quinoline-4-carbaldehyde 2a-d, dimedone or cyclohexane-1, 3dione 3a-b and (thio) urea $\mathbf{4 a - b}$ in the presence of concentrated $\mathrm{HCl}$ in ethanol. The structures of new compounds have been evaluated on the basis of elemental analysis, FT- IR, ${ }^{1} \mathrm{H}$ NMR and ${ }^{13} \mathrm{C}$ NMR spectral data. They have also been screened for their antimicrobial activities.
\end{abstract}

Keywords: Octahydroquinazolinones, tetrazolo[1,5-a]quinoline-4-carbaldehyde, Biginelli reaction, antimicrobial activity

\section{Introduction}

In recent years, dihydropyrimidinones (DHPMs) and their derivatives have occupied an important position in natural and synthetic organic chemistry, due mainly to their wide range of biological activities ${ }^{1}$, such as antibacterial, antiviral, antihypertensive, antitumor effects and calcium channel blockers. Scaffold decoration of DHPMs is highly important for creating structural diversity to produce "drug-like" molecules for biological screening. The synthesis of DHPMs was first reported by Biginelli in $1893^{2}$ and has been reviewed recently ${ }^{3}$. Improved procedures and new Biginelli-like scaffolds have been reported over the past decade and a variant of the Beginelli condensation has been described for its application to the total synthesis of bioactive guanidine alkaloids ${ }^{4}$. Basically, these methods are all similar in the use of different Lewis acid catalyst as well as protic acid under classical reflux ${ }^{5}$. Other studies have focused on the use of ionic liquids ${ }^{6}$, microwave irradiation ${ }^{7}$ and combinatorial chemistry ${ }^{8}$. The use of boron compounds $^{9}, \mathrm{TMSCl}^{10}$ and heterogeneous catalysts, such as tangstophosphoric acid ${ }^{11}$, Zeolite ${ }^{12}$, montmorillonite $^{13}$ and ion-exchange resins ${ }^{14}$ have been reported. However, to the best of our knowledge, there have been relatively few reports of the synthesis of fused DHPMs from cyclic 
$\beta$-diketones. More recently, the Biginelli reaction has been employed for the synthesis of octahydroquinazolinones, which used cyclic $\beta$-diketones instead of open-chain dicarbonyl compounds using concentrated $\mathrm{HCl}^{15}$ and $\mathrm{H}_{2} \mathrm{SO}_{4}{ }^{16}$ as the catalyst. Octahydroquinazolinone derivatives have attracted considerable attention since they exhibit potent antibacterial activity against Staphylococcus aureus, Escherichia coli, Pseudomonas aeruginosa ${ }^{17}$ and calcium antagonist activity ${ }^{18-19}$. Literature survey reveals that number of octahydroquinazolinone ${ }^{20}$ derivatives have been synthesized by Biginelli reaction conditions using various aldehyde but not a single reference have been found where tetrazolo[1,5-a]quinoline-4-carbaldehyde is used. In view of the above observation and in continuation of our work on biologically active heterocyclic compounds ${ }^{21-23}$, We now wish to report herein this heterocyclic aldehyde which is biologically active ${ }^{24-25}$ with a view to obtain more active heterocyclic systems containing two biologically active moieties tetrazoloquinolines ${ }^{26}$ and octahydroquinazolinone ${ }^{27-28}$.

\section{Results and Discussion}

The Octahydroquinazolinone 5a-p was synthesized by acid catalyzed condensation of 7(un)sub.tetrazolo[1,5-a]quinoline-4-carbaldehyde 2a-d, dimedone or cyclohexane-1,3-dione 3ab, (thio)urea 4a-b in ethanol by a modification of the Biginelli reaction in good yield(60-82\%). 7-(un)sub. tetrazolo[1,5-a]quinoline-4-carbaldehyde 2a-d was prepared by refluxing 2-chloro-3formyl quinoline, sodium azide and acetic acid in ethanol for 3-4 $\mathrm{hr}^{29}$. The required 2-chloro-3formyl quinoline was prepared by literature procedure ${ }^{30}$. The structure of compound 2a-d was confirmed by IR, ${ }^{1} \mathrm{H}$ NMR, ${ }^{13} \mathrm{C}$ NMR spectra. IR spectra of 2a exhibited absorptions at 3000, $3025 \mathrm{~cm}^{-1}$ for (aromatic C-H stretching), $1700 \mathrm{~cm}^{-1}$ for (carbonyl group) and $1625 \mathrm{~cm}^{-1}$ for $(\mathrm{C}=\mathrm{N}$ stretching). The ${ }^{1} \mathrm{H}$ NMR of compound 2a showed singlet at $\delta 10.45 \mathrm{ppm}$ for (CHO) and aromatic protons resonate as multiplets at $\delta 7.44-8.90 \mathrm{ppm}$. The ${ }^{13} \mathrm{C} \mathrm{NMR}$ spectrum of compound 2a showed signals at $\delta 113.74,118.52,122.89,124.77,125.08,126.54,140.43$, 145.32 for aromatic carbon, 159.34 for $(\mathrm{C}=\mathrm{N})$ and the carbonyl carbon was observed at $\delta 189.14$. The structure of compounds 5a-p was confirmed by IR, ${ }^{1} \mathrm{H}$ NMR, ${ }^{13} \mathrm{C}$ NMR and mass spectra. IR spectra of 5a exhibited absorptions at 3353, $3259 \mathrm{~cm}^{-1}$ for $(\mathrm{NH}), 3051 \mathrm{~cm}^{-1}$ for (aromatic C$\mathrm{H}$ stretching), $1701 \& 1666 \mathrm{~cm}^{-1}$ for (carbonyl group) and $1646 \mathrm{~cm}^{-1}$ for $(\mathrm{C}=\mathrm{N}$ stretching). The ${ }^{1} \mathrm{H}$ NMR of compound 5a showed singlet at $\delta 9.78$ and $7.70 \mathrm{ppm}$ for $(\mathrm{NH})$ proton, it also showed singlet at $\delta 0.81$ and $1.01 \mathrm{ppm}$ for $\left(\mathrm{CH}_{3}\right.$ of dimedone $)$ and $\delta 5.47 \mathrm{ppm}$ for $(\mathrm{CH})$ and a multiplet at $\delta 1.90-2.48$ for $\left(2 \mathrm{CH}_{2}\right)$. Aromatic protons resonate as multiplets at $\delta 7.80-8.62 \mathrm{ppm}$. The ${ }^{13} \mathrm{C}$ NMR spectrum of compound 5a showed signals at $\delta 27.30,29.18,32.67,39.35,50.29,52.26$ for aliphatic carbon, $\delta 151.58(\mathrm{C}=\mathrm{N})$ and $\delta 103.61,116.59,124.05,128.20,128.85,129.77,130.04$, $131.35,131.75,146.54$ for aromatic carbon and the carbonyl carbon was observed at $\delta 154.66$ and 193.32. The structure was further confirmed by its mass spectral studies. It gave molecular ion peak at $\mathrm{m} / \mathrm{z} 363(\mathrm{M}+1)$ corresponding to molecular formula $\mathrm{C}_{19} \mathrm{H}_{18} \mathrm{~N}_{6} \mathrm{O}_{2}$ (Scheme 1). Similarly, all these compounds were characterized on the basis of spectral studies. All the 
compounds were screened for their antibacterial and antifungal activities using ciprofloxacin, ampicillin and griseofulvin as standard drugs.<smiles></smiles>

$1 a-d$ 2a-d<smiles>[R]C1([R])CC(=O)CC(=O)C1</smiles>

3a-b<smiles>CCCCC(C)C(Cl)Cl</smiles>

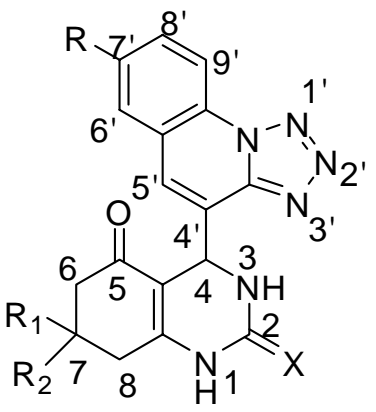<smiles>[X]C(N)=[W]</smiles>

$4 a-b$

$$
5 a-p
$$

$\begin{array}{llllllllll} & \mathrm{R} & \mathrm{R}_{1} & \mathrm{R}_{2} & \mathrm{X} & & \mathrm{R} & \mathrm{R}_{1} & \mathrm{R}_{2} & \mathrm{X} \\ \mathbf{5 a} & \mathrm{H} & \mathrm{CH}_{3} & \mathrm{CH}_{3} & \mathrm{O} & \mathbf{5 i} & \mathrm{H} & \mathrm{CH}_{3} & \mathrm{CH}_{3} & \mathrm{~S} \\ \mathbf{5 b} & \mathrm{CH}_{3} & \mathrm{CH}_{3} & \mathrm{CH}_{3} & \mathrm{O} & \mathbf{5 j} & \mathrm{CH}_{3} & \mathrm{CH}_{3} & \mathrm{CH}_{3} & \mathrm{~S} \\ \mathbf{5 c} & \mathrm{OCH}_{3} & \mathrm{CH}_{3} & \mathrm{CH}_{3} & \mathrm{O} & \mathbf{5 k} & \mathrm{OCH}_{3} & \mathrm{CH}_{3} & \mathrm{CH}_{3} & \mathrm{~S} \\ \mathbf{5 d} & \mathrm{Cl} & \mathrm{CH}_{3} & \mathrm{CH}_{3} & \mathrm{O} & \mathbf{5 l} & \mathrm{Cl} & \mathrm{CH}_{3} & \mathrm{CH}_{3} & \mathrm{~S} \\ \mathbf{5 e} & \mathrm{H} & \mathrm{H} & \mathrm{H} & \mathrm{O} & \mathbf{5 m} & \mathrm{H} & \mathrm{H} & \mathrm{H} & \mathrm{S} \\ \mathbf{5 f} & \mathrm{CH}_{3} & \mathrm{H} & \mathrm{H} & \mathrm{O} & \mathbf{5 n} & \mathrm{CH}_{3} & \mathrm{H} & \mathrm{H} & \mathrm{S} \\ \mathbf{5 g} & \mathrm{OCH}_{3} & \mathrm{H} & \mathrm{H} & \mathrm{O} & \mathbf{5 0} & \mathrm{OCH}_{3} & \mathrm{H} & \mathrm{H} & \mathrm{S} \\ \mathbf{5 h} & \mathrm{Cl} & \mathrm{H} & \mathrm{H} & \mathrm{O} & \mathbf{5 p} & \mathrm{Cl} & \mathrm{H} & \mathrm{H} & \mathrm{S}\end{array}$

Scheme 1. General synthetic procedure of octahydroquinazolinone 5a-p.

\section{Evaluation of antimicrobial activity}

The in vitro antimicrobial activity of compounds 5a-p were carried out against $24 \mathrm{hr}$ old cultures of three bacteria Escherichia coli as Gram-negative bacteria and Bacillus substilis and Staphylococcus aureus as Gram-positive bacteria and two fungi Aspergillus niger and Rhizopus oryzae by disc-diffusion method ${ }^{31-32}$.

Nutrient agar and potato dextrose agars were used to culture the bacteria and fungus respectively. The compounds were tested at a concentration of $1000 \mu \mathrm{g} / \mathrm{mL}$ in DMF solution. 
Ciprofloxacin, Ampicillin and Griseofulvin were used as standards for comparison of antibacterial and antifungal activities respectively. Inhibition was recorded by measuring the diameter of the inhibition zone at the end of $24 \mathrm{hr}$. for bacteria at $35{ }^{\circ} \mathrm{C}$ and $48 \mathrm{hr}$. for fungus at $28^{\circ} \mathrm{C}$. The protocols are summarized in (Table 1).

Table 1. Antimicrobial activity of the compounds 2a-d \& 5a-p

\begin{tabular}{|c|c|c|c|c|c|}
\hline \multirow[b]{2}{*}{ Compd. } & \multicolumn{3}{|c|}{$\begin{array}{c}\text { Antibacterial activity } \\
\text { Zone of inhibition in } \mathrm{mm}\end{array}$} & \multicolumn{2}{|c|}{$\begin{array}{l}\text { Antifungal activity } \\
\text { Zone of inhibition in } \mathrm{mm}\end{array}$} \\
\hline & E. coli & B. substilis & S. aureus & R. oryzae & A. niger \\
\hline $2 a$ & + & + & ++ & ++ & + \\
\hline $2 \mathbf{b}$ & + & ++ & + & ++ & + \\
\hline 2c & + & + & + & ++ & + \\
\hline $2 d$ & ++ & + & + & ++ & + \\
\hline $5 a$ & ++ & ++ & + & ++ & + \\
\hline $5 b$ & + & ++ & ++ & +++ & + \\
\hline $5 c$ & + & + & ++ & ++ & ++ \\
\hline $5 d$ & ++ & + & ++ & ++ & + \\
\hline $5 e$ & ++ & + & + & ++ & + \\
\hline $5 f$ & ++ & ++ & + & +++ & + \\
\hline $5 g$ & + & ++ & + & +++ & + \\
\hline $5 h$ & ++ & + & + & +++ & + \\
\hline $5 \mathbf{i}$ & + & + & ++ & ++ & + \\
\hline $5 \mathbf{j}$ & + & + & + & ++ & + \\
\hline $5 k$ & + & + & + & +++ & + \\
\hline 51 & ++ & + & ++ & +++ & + \\
\hline $5 m$ & + & + & +++ & +++ & + \\
\hline $5 n$ & + & + & ++ & ++ & + \\
\hline 50 & ++ & + & ++ & +++ & + \\
\hline $5 p$ & ++ & - & + & +++ & + \\
\hline Ciprofloxacin & +++ & +++ & +++ & NT & NT \\
\hline Ampicillin & +++ & +++ & +++ & NT & NT \\
\hline Griseofulvin & NT & NT & NT & +++ & +++ \\
\hline
\end{tabular}

$(-)=$ Inactive (inhibition zone $<10 \mathrm{~mm}$ );

$(+)=$ Slightly active (inhibition zone 10-14 mm);

$(++)=$ Moderate activity (inhibition zone 15-19 mm);

$(+++)=$ Good activity (inhibition zone $\geq 20 \mathrm{~mm}$ );

$\mathrm{NT}=$ not tested.

Control (DMF) (-) - No activity. 
The antifungal evaluation of the synthesized compounds revealed that among all the compounds $\mathbf{5 b}, \mathbf{5 f}, \mathbf{5 g}, \mathbf{5 h}, \mathbf{5 k}, \mathbf{5 l}, \mathbf{5 m}, \mathbf{5 0}$ and $\mathbf{5 p}$ showed good antifungal activity against fungal strain namely Rhizopus oryzae. Similarly compound 5a, 5c, 5d, 5e, 5g, 5i, 5j and 5 n against Rhizopus oryzae and compound 5c against Aspergillus niger showed moderate antifungal activity.

The antibacterial evaluation of the synthesized compounds revealed that among all the compounds $5 \mathrm{~m}$ showed good antibacterial activity against bacterial species namely Staphylococcus aureus. Compounds 5a, 5d, 5e, 5f, 5h, 5l, 50 and 5p showed moderate antibacterial activity against Gram-negative bacterial species Escherichia coli. Similarly compounds 5a, 5b, $\mathbf{5 f}$ and $\mathbf{5 g}$ against Gram-positive bacterial species Bacillus substilis and 5b, 5c, 5d, 5i, 5l, 5n and 50 against Gram-positive bacterial species Staphylococcus aureus showed moderate antibacterial activity. Compound 5p is inactive against Gram-positive bacterial species Bacillus substilis.

While remaining compounds of the series are slightly active against the Gram-positive, Gram-negative bacterial species and fungal species.

\section{Conclusion}

A convenient and efficient method for the direct synthesis of octahydroquinazolinone derivatives over concentrated $\mathrm{HCl}$ as the catalyst with good yield has been developed. This successful reaction expands the synthetic scope of the multicomponent Biginelli reaction. Further, the present procedure may be readily amenable to large-scale synthesis and the generation of combinatorial octahydroquinazolinones derivatives. It can be concluded from Table I that among all the compounds $\mathbf{5 b}, \mathbf{5 f}, \mathbf{5 g}, \mathbf{5 h}, \mathbf{5 k}, \mathbf{5 l}, \mathbf{5 m}, \mathbf{5 0}$ and $\mathbf{5 p}$ showed good antifungal activity against Rhizopus oryzae while compound $\mathbf{5 a}, \mathbf{5 c}, \mathbf{5 d}, \mathbf{5 e}, \mathbf{5 i}, \mathbf{5 j}$ and $\mathbf{5 n}$ against Rhizopus oryzae and $5 \mathrm{c}$ against Aspergillus niger showed moderate antifungal activity. Compound $5 \mathrm{~m}$ showed good antibacterial activity against Staphylococcus aureus. Compounds 5a, 5d, 5e, 5f, 5h, 5l, $\mathbf{5 0}$ and 5p against Escherichia coli, 5a, 5b, 5f and $\mathbf{5 g}$ against Bacillus substilis, 5b, 5c, 5d, 5i, 5l, 5n and 50 against Staphylococcus aureus showed moderate antibacterial activity while remaining compounds of the series are slightly active against the Gram-positive, Gram-negative bacterial species and fungal species.

\section{Experimental Section}

General Procedures. All the reagents were obtained commercially and used with further purification. All melting points were taken in open capillaries and are uncorrected. The monitoring of the progress of all reactions and homogeneity of the synthesized compounds was carried out by TLC. TLC was runned using TLC aluminum sheets silica gel $60 \mathrm{~F}_{254}$ (Merck). 
Elemental analysis (\% C, H, N) was carried out by Perkins Elmer $2400 \mathrm{CHN}$ elemental analyzer. IR spectra were recorded on a shimadzu FTIR 8401 spectrophotometer in KBR. ${ }^{1} \mathrm{H}$ NMR and ${ }^{13} \mathrm{C}$ NMR spectra were recorded on a Bruker Avance $400 \mathrm{MHz}$ spectrometer using solvent peak as internal standard. Mass spectra were scanned on a shimadzu LCMS 2010 spectrometer.

\section{General procedure for 7-(un)substituted-tetrazolo[1,5-a]quinoline-4-carbaldehyde (2a-d)}

2-chloro-3-formyl quinoline (0.005 moles), sodium azide (0.01 moles), acetic acid (1 mL) and ethanol $(10 \mathrm{~mL})$ were charged in R.B. flask with mechanical stirrer and condenser. The reaction mixture was slowly heated and refluxed for 3-4 hr. After the completion of reaction (checked by TLC), the product was filtered and washed with ethanol. The crude product was purified by leaching in volume ratio of chloroform and methanol $(10: 10 \mathrm{~mL})$ to obtain the pure solid sample. Tetrazolo[1,5-a]quinoline-4-carbaldehyde (2a). White solid, (82 \%), m.p. 245-246 ${ }^{\circ} \mathrm{C}$, Anal.Calcd for $\mathrm{C}_{10} \mathrm{H}_{6} \mathrm{~N}_{4} \mathrm{O}$ : C 60.60, H 3.05, N 28.27\% Found: C 60.70, H 2.93, N 28.38\%. IR $\left(\mathrm{KBr}, \mathrm{cm}^{-1}\right)$ : 3000, (ArC-H), $1700(\mathrm{C}=\mathrm{O}), 1570(\mathrm{C}=\mathrm{C}), 1625(\mathrm{C}=\mathrm{N}) .{ }^{1} \mathrm{H}$ NMR (400 MHz, DMSO-d $\left.d_{6}\right): \delta 7.44-8.90(\mathrm{~m}, 5 \mathrm{H}, \mathrm{Ar}-\mathrm{H}), 10.45$ (s, 1H, CHO), ${ }^{13} \mathrm{C}$ NMR $\left(400 \mathrm{MHz}, \mathrm{DMSO}-d_{6}\right) \delta$ : $113.74,118.52,122.89,124.77,125.08,126.54,140.43($ Ar-C), $145.32(\mathrm{C}-\mathrm{N}), 159.34(\mathrm{C}=\mathrm{N})$, $189.14(\mathrm{C}=\mathrm{O})$.

7-methyltetrazolo[1,5-a]quinoline-4-carbaldehyde (2b). White solid, (85 \%), m.p. 258-260 ${ }^{\circ} \mathrm{C}$, Anal.Calcd for $\mathrm{C}_{11} \mathrm{H}_{8} \mathrm{~N}_{4} \mathrm{O}: \mathrm{C}$ 62.25, H 3.80, N 26.40\% Found: C 62.34, H 3.95, N 26.27\%. IR ( $\left.\mathrm{KBr}, \mathrm{cm}^{-1}\right): 3020(\mathrm{ArC}-\mathrm{H}), 2910\left(\mathrm{CH}_{3}\right), 1710(\mathrm{C}=\mathrm{O}), 1580(\mathrm{C}=\mathrm{C}), 1620(\mathrm{C}=\mathrm{N}) .{ }^{1} \mathrm{H}$ NMR (400 MHz, DMSO-d $\left.)_{6}\right): \delta 2.35\left(\mathrm{~s}, 3 \mathrm{H}, \mathrm{CH}_{3}\right), 7.75-8.96(\mathrm{~m}, 4 \mathrm{H}, \mathrm{Ar}-\mathrm{H}), 10.40(\mathrm{~s}, 1 \mathrm{H}, \mathrm{CHO}),{ }^{13} \mathrm{C}$ NMR (400 MHz, DMSO-d 6 ) $\delta: 21.80\left(\mathrm{CH}_{3}\right), 113.64,118.37,122.19,124.22,124.90,126.54$, $139.55($ Ar-C), $145.12(\mathrm{C}-\mathrm{N}), 159.37(\mathrm{C}=\mathrm{N}), 189.14(\mathrm{C}=\mathrm{O})$.

7-methoxytetrazolo[1,5-a]quinoline-4-carbaldehyde (2c). White solid, (80 \%), m.p. 236-237 ${ }^{\circ} \mathrm{C}$, Anal.Calcd for $\mathrm{C}_{11} \mathrm{H}_{8} \mathrm{~N}_{4} \mathrm{O}_{2}$ : C 57.89, H 3.53, N 24.55\% Found: C 57.77, H 3.64, N 24.67\%. IR $\left(\mathrm{KBr}, \mathrm{cm}^{-1}\right): 3015(\mathrm{ArC}-\mathrm{H}), 1260 \& 1050\left(\mathrm{OCH}_{3}\right), 1690(\mathrm{C}=\mathrm{O}), 1590(\mathrm{C}=\mathrm{C}), 1610(\mathrm{C}=\mathrm{N}) .{ }^{1} \mathrm{H}$ NMR (400 MHz, DMSO-d $)): \delta 3.97\left(\mathrm{~s}, 3 \mathrm{H}, \mathrm{OCH}_{3}\right), 7.71-8.91(\mathrm{~m}, 4 \mathrm{H}, \mathrm{Ar}-\mathrm{H}), 10.43(\mathrm{~s}, 1 \mathrm{H}$, $\mathrm{CHO}),{ }^{13} \mathrm{C}$ NMR (400 MHz, DMSO-d 6$) \delta: 56.59\left(\mathrm{OCH}_{3}\right), 112.84,118.48,122.09,124.07$, 124.88, 126.64, $139.43($ Ar-C), $145.02(\mathrm{C}-\mathrm{N}), 159.21(\mathrm{C}=\mathrm{N}), 189.04(\mathrm{C}=\mathrm{O})$.

7-chlorotetrazolo[1,5-a]quinoline-4-carbaldehyde (2d). Off-white solid, (75 \%), m.p. 220-221 ${ }^{\circ} \mathrm{C}$, Anal.Calcd for $\mathrm{C}_{10} \mathrm{H}_{5} \mathrm{~N}_{4} \mathrm{O}: \mathrm{C} 51.63, \mathrm{H} 2.16$, N 24.08\% Found: C 51.72, H 2.25, N 23.95\%. IR $\left(\mathrm{KBr}, \mathrm{cm}^{-1}\right): 2990(\mathrm{ArC}-\mathrm{H}), 1695(\mathrm{C}=\mathrm{O}), 1585(\mathrm{C}=\mathrm{C}), 1615(\mathrm{C}=\mathrm{N}), 750(\mathrm{C}-\mathrm{Cl}) .{ }^{1} \mathrm{H}$ NMR (400 MHz, DMSO-d $\left.d_{6}\right): \delta 7.80-8.81(\mathrm{~m}, 4 \mathrm{H}, \mathrm{Ar}-\mathrm{H}), 10.52(\mathrm{~s}, 1 \mathrm{H}, \mathrm{CHO}),{ }^{13} \mathrm{C} \mathrm{NMR}(400 \mathrm{MHz}$, DMSO-d $\left.{ }_{6}\right) \delta: 114.14,118.23,122.79,124.77,125.45,126.28,140.65$ (Ar-C), $145.82(\mathrm{C}-\mathrm{N})$, $159.25(\mathrm{C}=\mathrm{N}), 189.28(\mathrm{C}=\mathrm{O})$.

General procedure for 7,7-dimethyl-4-(tetrazolo[1,5-a]quinolin-4-yl)-3,4,7,8tetrahydroquinazoline-2,5(1H, 6H)-dione (5a-p)

Tetrazolo(1,5-a)quinoline-4-carbaldehyde (0.0025 mole), dimedone or cyclohexane-1,3-dione (0.0025 mole), (thio)urea $(0.0025 \mathrm{~mol})$, conc. $\mathrm{HCl}(0.1 \mathrm{~mL})$ and ethanol $(15 \mathrm{~mL})$ were charged in 
R.B. flask with mechanical stirrer and condenser. The reaction mixture was slowly heated and refluxed for $5 \mathrm{hr}$. After the completion of reaction (checked by TLC), the product was filtered and washed with ethanol. The crude product was purified by leaching in volume ratio of chloroform and methanol $(10: 10 \mathrm{~mL})$ to obtain the pure solid sample.

7,7-dimethyl-4-(tetrazolo[1,5-a]quinolin-4-yl)-3,4,7,8-tetrahydroquinazoline-2,5(1H, $6 \mathrm{H})$ dione (5a). White solid, (82 \%), m.p. 290-292 ${ }^{\circ} \mathrm{C}$, Anal.Calcd for $\mathrm{C}_{19} \mathrm{H}_{18} \mathrm{~N}_{6} \mathrm{O}_{2}$ : C 62.97, H 5.00, N 23.19\% Found: C 63.11, H 4.89, N 23.34\%. IR (KBr, cm $\left.{ }^{-1}\right): 3353,3259$ (2NH), 3051 (ArC$\mathrm{H}), 1701(\mathrm{C}=\mathrm{O}), 1666(\mathrm{C}=\mathrm{O}), 1614(\mathrm{C}=\mathrm{C}), 1646(\mathrm{C}=\mathrm{N}) .{ }^{1} \mathrm{H}$ NMR (400 MHz, DMSO-d $)$ : $\delta$ $0.81\left(\mathrm{~s}, 3 \mathrm{H}, \mathrm{CH}_{3}\right), 1.01\left(\mathrm{~s}, 3 \mathrm{H}, \mathrm{CH}_{3}\right), 1.90-2.48\left(\mathrm{~m}, 4 \mathrm{H}, 2 \mathrm{CH}_{2}\right), 5.74(\mathrm{~s}, 1 \mathrm{H}, \mathrm{CH}), 7.70(\mathrm{~s}, 1 \mathrm{H}$, $\mathrm{NH}), 7.80-8.62(\mathrm{~m}, 5 \mathrm{H}, \mathrm{Ar}-\mathrm{H}), 9.78(\mathrm{~s}, 1 \mathrm{H}, \mathrm{NH}),{ }^{13} \mathrm{C}$ NMR (400 MHz, DMSO-d 6 ) $\delta: 27.30$ $\left(\mathrm{CH}_{3}\right), 29.18\left(\mathrm{CH}_{3}\right), 32.67(\mathrm{C}), 39.35\left(\mathrm{CH}_{2}\right), 50.29\left(\mathrm{CH}_{2}\right), 52.26(\mathrm{CH}), 103.61,116.59,124.05$, 128.20, 128.85, 129.77, 130.04, 131.35, 131.75, $146.54(\mathrm{Ar}-\mathrm{C}), 151.58(\mathrm{C}=\mathrm{N}), 154.66(\mathrm{C}=\mathrm{O})$, $193.32(\mathrm{C}=\mathrm{O}), \mathrm{MS}:(\mathrm{M}+1) 363$.

\section{7,7-dimethyl-4-(7-methyltetrazolo[1,5-a]quinolin-4-yl)-3,4,7,8-tetrahydroquinazoline-}

2,5(1H, 6H)-dione (5b). White solid, (73 \%), m.p. $>300{ }^{\circ} \mathrm{C}$, Anal. Calcd. for $\mathrm{C}_{20} \mathrm{H}_{20} \mathrm{~N}_{6} \mathrm{O}_{2}: \mathrm{C}$ 63.81, H 5.35, N 22.32\% Found: C 63.69, H 5.51, N 22.42\%. IR (KBr, cm $\left.{ }^{-1}\right): 3310,3265(2 \mathrm{NH})$, 3020 (ArC-H), $2920\left(\mathrm{CH}_{3}\right), 1718(\mathrm{C}=\mathrm{O}), 1650(\mathrm{C}=\mathrm{O}), 1604(\mathrm{C}=\mathrm{C}), 1640(\mathrm{C}=\mathrm{N}) .{ }^{1} \mathrm{H}$ NMR $(400$ $\left.\mathrm{MHz}, \mathrm{DMSO}-\mathrm{d}_{6}\right): \delta 0.81\left(\mathrm{~s}, 3 \mathrm{H}, \mathrm{CH}_{3}\right), 0.99\left(\mathrm{~s}, 3 \mathrm{H}, \mathrm{CH}_{3}\right), 2.34\left(\mathrm{~s}, 3 \mathrm{H}, \mathrm{CH}_{3}\right), 1.88-2.45(\mathrm{~m}, 4 \mathrm{H}$, $\left.2 \mathrm{CH}_{2}\right), \quad 5.70(\mathrm{~s}, 1 \mathrm{H}, \mathrm{CH}), 7.77(\mathrm{~s}, 1 \mathrm{H}, \mathrm{NH}), 7.60-8.82(\mathrm{~m}, 4 \mathrm{H}, \mathrm{Ar}-\mathrm{H}), 9.56(\mathrm{~s}, 1 \mathrm{H}, \mathrm{NH}),{ }^{13} \mathrm{C}$ NMR (400 MHz, DMSO-d $) \delta: 21.70\left(\mathrm{CH}_{3}\right), 27.20\left(\mathrm{CH}_{3}\right), 29.20\left(\mathrm{CH}_{3}\right), 32.71(\mathrm{C}), 39.26\left(\mathrm{CH}_{2}\right)$, $50.80\left(\mathrm{CH}_{2}\right), 52.19(\mathrm{CH}), 104.10,116.50,124.00,128.40,129.00,129.90,130.03,131.24$, 131.55, $146.72($ Ar-C), $151.40(\mathrm{C}=\mathrm{N}), 154.18(\mathrm{C}=\mathrm{O}), 193.28(\mathrm{C}=\mathrm{O})$.

7,7-dimethyl-4-(7-methoxytetrazolo[1,5-a]quinolin-4-yl)-3,4,7,8-tetrahydroquinazoline2,5(1H, 6H)-dione (5c). White solid, (64 \%), m.p. 298-300 ${ }^{\circ} \mathrm{C}$, Anal. Calcd. for $\mathrm{C}_{20} \mathrm{H}_{20} \mathrm{~N}_{6} \mathrm{O}_{3}: \mathrm{C}$ 61.21, H 5.13, N 21.41\% Found: C 61.08, H 5.25, N 21.55\%. IR (KBr, cm $\left.{ }^{-1}\right): 3431,3281(2 \mathrm{NH})$, $3077\left(\right.$ ArC-H), $1721(\mathrm{C}=\mathrm{O}), 1627(\mathrm{C}=\mathrm{O}), 1524(\mathrm{C}=\mathrm{C}), 1579(\mathrm{C}=\mathrm{N}), 1236 \& 1046\left(\mathrm{OCH}_{3}\right) .{ }^{1} \mathrm{H}$ NMR (400 MHz, DMSO-d $\left.d_{6}\right): \delta 0.81\left(\mathrm{~s}, 3 \mathrm{H}, \mathrm{CH}_{3}\right), 1.01\left(\mathrm{~s}, 3 \mathrm{H}, \mathrm{CH}_{3}\right), 3.92\left(\mathrm{~s}, 3 \mathrm{H}, \mathrm{OCH}_{3}\right), 1.92-$ $2.47\left(\mathrm{~m}, 4 \mathrm{H}, 2 \mathrm{CH}_{2}\right), 5.73(\mathrm{~s}, 1 \mathrm{H}, \mathrm{CH}), 7.72(\mathrm{~s}, 1 \mathrm{H}, \mathrm{NH}), 7.50-8.49(\mathrm{~m}, 4 \mathrm{H}, \mathrm{Ar}-\mathrm{H}), 9.78(\mathrm{~s}, 1 \mathrm{H}$, $\mathrm{NH}),{ }^{13} \mathrm{C}$ NMR (400 MHz, DMSO-d $) \delta: 27.39\left(\mathrm{CH}_{3}\right), 29.10\left(\mathrm{CH}_{3}\right), 32.68(\mathrm{C}), 39.35\left(\mathrm{CH}_{2}\right)$, $50.29\left(\mathrm{CH}_{2}\right), 52.22(\mathrm{CH}), 56.36\left(\mathrm{OCH}_{3}\right), 103.68,110.17,118.01,120.98,124.23,125.61$, 128.59, 130.92, 145.87, $151.59($ Ar-C), $154.62(\mathrm{C}=\mathrm{N}), 159.08(\mathrm{C}=\mathrm{O}), 193.31(\mathrm{C}=\mathrm{O})$.

\section{7,7-dimethyl-4-(7-chlorotetrazolo[1,5-a]quinolin-4-yl)-3,4,7,8-tetrahydroquinazoline-}

2,5(1H, 6H)-dione (5d). Off-white solid, (60 \%), m.p. $>300{ }^{\circ} \mathrm{C}$, Anal. Calcd. for $\mathrm{C}_{19} \mathrm{H}_{17} \mathrm{ClN}_{6} \mathrm{O}_{2}$ : C 57.50, H 4.31, N 21.17\% Found: C 57.63, H 4.46, N 21.03\%. IR (KBr, cm $\left.{ }^{-1}\right): 3320,3260$ (2NH), $2980\left(\right.$ ArC-H), $1694(\mathrm{C}=\mathrm{O}), 1645(\mathrm{C}=\mathrm{O}), 1582(\mathrm{C}=\mathrm{C}), 1645(\mathrm{C}=\mathrm{N}), 745(\mathrm{C}-\mathrm{Cl}) .{ }^{1} \mathrm{H}$ NMR (400 MHz, DMSO-d $\left.)_{6}\right): \delta 0.84\left(\mathrm{~s}, 3 \mathrm{H}, \mathrm{CH}_{3}\right), 1.00\left(\mathrm{~s}, 3 \mathrm{H}, \mathrm{CH}_{3}\right), 1.90-2.38\left(\mathrm{~m}, 4 \mathrm{H}, 2 \mathrm{CH}_{2}\right)$, $5.72(\mathrm{~s}, 1 \mathrm{H}, \mathrm{CH}), 7.61(\mathrm{~s}, 1 \mathrm{H}, \mathrm{NH}), 7.62-7.79(\mathrm{~m}, 4 \mathrm{H}, \mathrm{Ar}-\mathrm{H}), 9.66(\mathrm{~s}, 1 \mathrm{H}, \mathrm{NH}) .{ }^{13} \mathrm{C}$ NMR $(400$ MHz, DMSO-d $)_{6} \delta: 27.33\left(\mathrm{CH}_{3}\right), 29.10\left(\mathrm{CH}_{3}\right), 32.49(\mathrm{C}), 39.30\left(\mathrm{CH}_{2}\right), 50.15\left(\mathrm{CH}_{2}\right), 52.34$ $(\mathrm{CH}), 103.48,116.40,124.17,128.14,128.70,129.62,129.94,131.18,131.48,146.12$ (Ar-C), $151.27(\mathrm{C}=\mathrm{N}), 154.52(\mathrm{C}=\mathrm{O}), 193.38(\mathrm{C}=\mathrm{O})$. 
4-(tetrazolo[1,5-a]quinolin-4-yl)-3,4,7,8-tetrahydroquinazoline-2,5(1H, 6H)-dione (5e). Offwhite solid, (80 \%), m.p. 283-285 ${ }^{\circ} \mathrm{C}$, Anal. Calcd. for $\mathrm{C}_{17} \mathrm{H}_{14} \mathrm{~N}_{6} \mathrm{O}_{2}$ : C 61.07, H 4.22, N 25.13\% Found: C 61.21, H 4.34, N 25.00\%. IR (KBr, cm $\left.{ }^{-1}\right)$ : 3279, 3223 (2NH), 2952 (ArC-H), 1697 $(\mathrm{C}=\mathrm{O}), 1663(\mathrm{C}=\mathrm{O}), 1537(\mathrm{C}=\mathrm{C}), 1645(\mathrm{C}=\mathrm{N}) .{ }^{1} \mathrm{H}$ NMR $\left.(400 \mathrm{MHz} \text { DMSO-d })_{6}\right): \delta$ 1.85-2.27 (m, $\left.6 \mathrm{H}, 3 \mathrm{CH}_{2}\right), 5.77(\mathrm{~s}, 1 \mathrm{H}, \mathrm{CH}), 7.71(\mathrm{~s}, 1 \mathrm{H}, \mathrm{NH}), 7.77-8.60(\mathrm{~m}, 5 \mathrm{H}, \mathrm{Ar}-\mathrm{H}), 9.79(\mathrm{~s}, 1 \mathrm{H}, \mathrm{NH}) .{ }^{13} \mathrm{C}$ NMR (400 MHz, DMSO-d $)_{6} \delta: 21.17\left(\mathrm{CH}_{2}\right), 26.57\left(\mathrm{CH}_{2}\right), 36.71\left(\mathrm{CH}_{2}\right), 51.57(\mathrm{CH}), 104.82$, $116.57,124.10,128.22,128.80,129.78,130.08,130.99,131.74,146.66($ Ar-C $), 151.52(\mathrm{C}=\mathrm{N})$, $156.58(\mathrm{C}=\mathrm{O}), 193.68(\mathrm{C}=\mathrm{O}), \mathrm{MS}:(\mathrm{M}+1) 335$.

4-(7-methyltetrazolo[1,5-a]quinolin-4-yl)-3,4,7,8-tetrahydroquinazoline-2,5(1H, $6 \mathrm{H})$-dione (5f). Off-white solid, (65 \%), m.p. $291-293{ }^{\circ} \mathrm{C}$, Anal. Calcd. for $\mathrm{C}_{18} \mathrm{H}_{16} \mathrm{~N}_{6} \mathrm{O}_{2}$ : C 62.06, H 4.62, $\mathrm{N}$ 24.12\% Found: C 62.16, H 4.48, N 24.23\%. IR (KBr, cm $\left.{ }^{-1}\right): 3310,3250(2 \mathrm{NH}), 3015$ (ArC-H), $2910\left(\mathrm{CH}_{3}\right), 1682(\mathrm{C}=\mathrm{O}), 1650(\mathrm{C}=\mathrm{O}), 1600(\mathrm{C}=\mathrm{C}), 1640(\mathrm{C}=\mathrm{N}) .{ }^{1} \mathrm{H}$ NMR $(400 \mathrm{MHz}, \mathrm{DMSO}-$ $\left.d_{6}\right): \delta 1.84-1.94\left(\mathrm{~m}, 6 \mathrm{H}, 3 \mathrm{CH}_{2}\right), 3.34\left(\mathrm{~s}, 3 \mathrm{H}, \mathrm{CH}_{3}\right), 5.76(\mathrm{~s}, 1 \mathrm{H}, \mathrm{CH}), 7.71(\mathrm{~s}, 1 \mathrm{H}, \mathrm{NH}), 7.71-8.45$ (m, 4H, Ar-H), $9.77(\mathrm{~s}, 1 \mathrm{H}, \mathrm{NH}) .{ }^{13} \mathrm{C} \mathrm{NMR}\left(400 \mathrm{MHz}, \mathrm{DMSO}-d_{6}\right) \delta: 21.18\left(\mathrm{CH}_{2}\right), 21.31\left(\mathrm{CH}_{2}\right)$, $26.58\left(\mathrm{CH}_{2}\right), 36.71\left(\mathrm{CH}_{3}\right), 51.41(\mathrm{CH}), 104.90,116.30,124.12,127.87,128.14,129.38,130.67$, 132.90, 138.54, $146.46($ Ar-C), $151.54(\mathrm{C}=\mathrm{N}), 156.59(\mathrm{C}=\mathrm{O}), 193.66(\mathrm{C}=\mathrm{O})$.

4-(7-methoxytetrazolo[1,5-a]quinolin-4-yl)-3,4,7,8-tetrahydroquinazoline-2,5(1H, $6 \mathrm{H})$-dione (5g). White solid, (73 \%), m.p. 288-290 ${ }^{\circ} \mathrm{C}$, Anal. Calcd. for $\mathrm{C}_{18} \mathrm{H}_{16} \mathrm{~N}_{6} \mathrm{O}_{3}:$ C 59.36, H 4.42, N 23.06\% Found: C 59.27, H 4.58, N 23.18\%. IR (KBr, cm $\left.{ }^{-1}\right): 3280,3235$ (2NH), 2985 (ArC-H), $1668(\mathrm{C}=\mathrm{O}), 1640(\mathrm{C}=\mathrm{O}), 1590(\mathrm{C}=\mathrm{C}), 1642(\mathrm{C}=\mathrm{N}), 1245 \& 1050\left(\mathrm{OCH}_{3}\right) .{ }^{1} \mathrm{H}$ NMR $(400 \mathrm{MHz}$, DMSO-d $\left.d_{6}\right): \delta 1.90-2.38\left(\mathrm{~m}, 6 \mathrm{H}, 3 \mathrm{CH}_{2}\right), 3.85\left(\mathrm{~s}, 3 \mathrm{H}, \mathrm{OCH}_{3}\right), 5.70(\mathrm{~s}, 1 \mathrm{H}, \mathrm{CH}), 7.69(\mathrm{~s}, 1 \mathrm{H}, \mathrm{NH})$, 7.78-8.56 (m, 4H, Ar-H), $9.73(\mathrm{~s}, 1 \mathrm{H}, \mathrm{NH}) .{ }^{13} \mathrm{C}$ NMR (400 MHz, DMSO-d 6$) \delta: 21.18\left(\mathrm{CH}_{2}\right)$, $22.23\left(\mathrm{CH}_{2}\right), 28.11\left(\mathrm{CH}_{2}\right), 36.25(\mathrm{CH}), 55.81\left(\mathrm{OCH}_{3}\right), 104.78,116.47,124.35,127.90,128.10$, 129.42, 130.50, 132.75, 138.48, $146.28($ Ar-C), $151.70(\mathrm{C}=\mathrm{N}), 156.39(\mathrm{C}=\mathrm{O}), 193.56(\mathrm{C}=\mathrm{O})$.

4-(7-chlorotetrazolo[1,5-a]quinolin-4-yl)-3,4,7,8-tetrahydroquinazoline-2,5(1H, 6H)-dione (5h). Grey solid, (62 \%), m.p. 296-298 ${ }^{\circ} \mathrm{C}$, Anal. Calcd. for $\mathrm{C}_{17} \mathrm{H}_{13} \mathrm{ClN}_{6} \mathrm{O}_{2}$ : C 55.36, H 3.55, N 22.78\% Found: C 55.51, H 3.42, N 22.92\%. IR (KBr, cm $\left.{ }^{-1}\right): 3320,3260(2 \mathrm{NH}), 3010$ (ArC-H), $1684(\mathrm{C}=\mathrm{O}), 1640(\mathrm{C}=\mathrm{O}), 1597(\mathrm{C}=\mathrm{C}), 1650(\mathrm{C}=\mathrm{N}), 750(\mathrm{C}-\mathrm{Cl}) .{ }^{1} \mathrm{H}$ NMR (400 MHz, DMSO$\left.d_{6}\right): \delta 1.87-2.25\left(\mathrm{~m}, 6 \mathrm{H}, 3 \mathrm{CH}_{2}\right), 5.60(\mathrm{~s}, 1 \mathrm{H}, \mathrm{CH}), 7.75(\mathrm{~s}, 1 \mathrm{H}, \mathrm{NH}), 7.68-8.60(\mathrm{~m}, 4 \mathrm{H}, \mathrm{Ar}-\mathrm{H})$, $9.82(\mathrm{~s}, 1 \mathrm{H}, \mathrm{NH}) .{ }^{13} \mathrm{C}$ NMR $\left(400 \mathrm{MHz}, \mathrm{DMSO}-d_{6}\right) \delta: 21.10\left(\mathrm{CH}_{2}\right), 26.35\left(\mathrm{CH}_{2}\right), 36.61\left(\mathrm{CH}_{2}\right)$, $51.64(\mathrm{CH}), 104.88,116.49,124.17,128.12,128.92,129.75,130.00,130.88,131.86,146.72$ (Ar-C), $151.48(\mathrm{C}=\mathrm{N}), 156.46(\mathrm{C}=\mathrm{O}), 193.62(\mathrm{C}=\mathrm{O})$.

7,7-dimethyl-4-(tetrazolo[1,5-a]quinolin-4-yl)-2-thioxo-1,2,3,4,7,8-hexahydroquinazolin-

5(6H)-one (5i). Off-white solid, (79 \%), m.p. 299-300 ${ }^{\circ} \mathrm{C}$, Anal. Calcd. for $\mathrm{C}_{19} \mathrm{H}_{18} \mathrm{~N}_{6} \mathrm{OS}$ : C 60.30, H 4.79, N 22.20\% Found: C 60.44, H 4.95, N 22.04\%. IR (KBr, cm $\left.{ }^{-1}\right): 3292,3098$ (2NH), $3017\left(\right.$ ArC-H), $1712(\mathrm{C}=\mathrm{O}), 1623(\mathrm{C}=\mathrm{C}), 1671(\mathrm{C}=\mathrm{N}), 1191(\mathrm{C}=\mathrm{S}) .{ }^{1} \mathrm{H}$ NMR $(400$ MHz, DMSO-d $\left.)_{6}\right): \delta 0.78\left(\mathrm{~s}, 3 \mathrm{H}, \mathrm{CH}_{3}\right), 0.98\left(\mathrm{~s}, 3 \mathrm{H}, \mathrm{CH}_{3}\right), 1.86-2.38\left(\mathrm{~m}, 4 \mathrm{H}, 2 \mathrm{CH}_{2}\right), 5.78(\mathrm{~s}, 1 \mathrm{H}$, $\mathrm{CH}), 9.45$ (s, 1H, NH), 7.82-8.57 (m, 5H, Ar-H), $10.76(\mathrm{~s}, 1 \mathrm{H}, \mathrm{NH}) .{ }^{13} \mathrm{C} \mathrm{NMR} \mathrm{(400} \mathrm{MHz,}$ DMSO-d $\left.d_{6}\right) \delta: 27.30\left(\mathrm{CH}_{3}\right), 29.18\left(\mathrm{CH}_{3}\right), 32.67(\mathrm{C}), 39.15\left(\mathrm{CH}_{2}\right), 50.29\left(\mathrm{CH}_{2}\right), 52.26(\mathrm{CH})$, 
103.61, 116.59, 124.05, 128.20, 128.85, 129.77, 130.04, 131.35, 131.75, 146.54 (Ar-C), 151.58 $(\mathrm{C}=\mathrm{N}), 175.66(\mathrm{C}=\mathrm{S}), 193.32(\mathrm{C}=\mathrm{O})$.

\section{7,7-dimethyl-4-(7-methyltetrazolo[1,5-a]quinolin-4-yl)-2-thioxo-1,2,3,4,7,8-}

hexahydroquinazolin-5(6H)-one (5j). White solid, (65\%), m.p. $>300{ }^{\circ} \mathrm{C}$, Anal. Calcd. for $\mathrm{C}_{20} \mathrm{H}_{20} \mathrm{~N}_{6} \mathrm{OS}$ : C 61.20, H 5.13, N 21.41\% Found: C 61.05, H 5.24, N 21.58\%. IR (KBr, cm $\left.{ }^{-1}\right)$ : 3180, $3120(2 \mathrm{NH}), 3000(\mathrm{ArC}-\mathrm{H}), 1650(\mathrm{C}=\mathrm{O}), 2930\left(\mathrm{CH}_{3}\right), 1580(\mathrm{C}=\mathrm{C}), 1570(\mathrm{C}=\mathrm{N}), 1200$ $(\mathrm{C}=\mathrm{S}) .{ }^{1} \mathrm{H}$ NMR $\left(400 \mathrm{MHz}, \mathrm{DMSO}-d_{6}\right): \delta 0.82\left(\mathrm{~s}, 3 \mathrm{H}, \mathrm{CH}_{3}\right), 1.02\left(\mathrm{~s}, 3 \mathrm{H}, \mathrm{CH}_{3}\right), 3.34(\mathrm{~s}, 3 \mathrm{H}$, $\left.\mathrm{CH}_{3}\right), 1.93-2.47$ (m, 4H, 2CH$), 5.75(\mathrm{~s}, 1 \mathrm{H}, \mathrm{CH}), 9.57$ (s, 1H, NH), 7.76-8.45 (m, 4H, Ar-H), 10.79 (s, 1H, NH). ${ }^{13} \mathrm{C}$ NMR $\left(400 \mathrm{MHz}, \mathrm{DMSO}-d_{6}\right) \delta: 21.31\left(\mathrm{CH}_{3}\right), 27.28\left(\mathrm{CH}_{3}\right), 29.23\left(\mathrm{CH}_{3}\right)$, $32.63(\mathrm{C}), 39.12\left(\mathrm{CH}_{2}\right), 50.29\left(\mathrm{CH}_{2}\right), 52.10(\mathrm{CH}), 104.97,116.34,123.94,127.00,127.95$, 129.40, 131.94, 133.19, 138.72, $148.26($ Ar-C), $150.65(\mathrm{C}=\mathrm{N}), 175.15(\mathrm{C}=\mathrm{S}), 194.06(\mathrm{C}=\mathrm{O})$.

7,7-dimethyl-4-(7-methoxytetrazolo[1,5-a]quinolin-4-yl)-2-thioxo-1,2,3,4,7,8-

hexahydroquinazolin-5(6H)-one (5k). Off-white solid, (72\%), m.p. $>300{ }^{\circ} \mathrm{C}$, Anal. Calcd. for $\mathrm{C}_{20} \mathrm{H}_{20} \mathrm{~N}_{6} \mathrm{O}_{2} \mathrm{~S}$ : C 58.80, H 4.93, N 20.57\% Found C 58.94, H 5.04, N 20.47\%. IR (KBr, cm $\left.{ }^{-1}\right)$ : 3276, $3230(2 \mathrm{NH}), 2995(\mathrm{ArC}-\mathrm{H}), 1660(\mathrm{C}=\mathrm{O}), 1570(\mathrm{C}=\mathrm{C}), 1635(\mathrm{C}=\mathrm{N}), 1230 \& 1040\left(\mathrm{OCH}_{3}\right)$, $1190(\mathrm{C}=\mathrm{S}) .{ }^{1} \mathrm{H}$ NMR (400 MHz, DMSO-d $): \delta 0.79\left(\mathrm{~s}, 3 \mathrm{H}, \mathrm{CH}_{3}\right), 0.98\left(\mathrm{~s}, 3 \mathrm{H}, \mathrm{CH}_{3}\right), 1.80-1.94$ $\left(\mathrm{m}, 4 \mathrm{H}, 2 \mathrm{CH}_{2}\right), 3.92\left(\mathrm{~s}, 3 \mathrm{H}, \mathrm{OCH}_{3}\right), 5.76(\mathrm{~s}, 1 \mathrm{H}, \mathrm{CH}), 9.45(\mathrm{~s}, 1 \mathrm{H}, \mathrm{NH}), 7.78-8.56(\mathrm{~m}, 4 \mathrm{H}, \mathrm{Ar}-$ $\mathrm{H}), 10.60(\mathrm{~s}, 1 \mathrm{H}, \mathrm{NH}) .{ }^{13} \mathrm{C} \mathrm{NMR}\left(400 \mathrm{MHz}, \mathrm{DMSO}-d_{6}\right) \delta: 27.34\left(\mathrm{CH}_{3}\right), 29.28\left(\mathrm{CH}_{3}\right), 33.45(\mathrm{C})$, $39.23\left(\mathrm{CH}_{2}\right), 50.60\left(\mathrm{CH}_{2}\right), 52.23(\mathrm{CH}), 55.90\left(\mathrm{OCH}_{3}\right), 105.88,116.67,124.65,127.78,128.35$, 129.43, 130.59, 132.65, 138.58, $146.22($ Ar-C), $151.82(\mathrm{C}=\mathrm{N}), 175.44(\mathrm{C}=\mathrm{S}), 193.67(\mathrm{C}=\mathrm{O})$.

7,7-dimethyl-4-(7-chlorotetrazolo[1,5-a]quinolin-4-yl)-2-thioxo-1,2,3,4,7,8-

hexahydroquinazolin-5(6H)-one (5l). Off-white solid, (64\%), m.p. $>300{ }^{\circ} \mathrm{C}$, Anal. Calcd. for $\mathrm{C}_{19} \mathrm{H}_{17} \mathrm{ClN}_{6} \mathrm{OS}$ : C 55.27, H 4.15, N 20.35\% Found C 55.40, H 3.98, N 20.47\%. IR (KBr, cm $\left.{ }^{-1}\right)$ : 3247, $3210(2 \mathrm{NH}), 3015(\mathrm{ArC}-\mathrm{H}), 1668(\mathrm{C}=\mathrm{O}), 1587(\mathrm{C}=\mathrm{C}), 1640(\mathrm{C}=\mathrm{N}), 735(\mathrm{C}-\mathrm{Cl}), 1185$ $(\mathrm{C}=\mathrm{S}) .{ }^{1} \mathrm{H}$ NMR $\left(400 \mathrm{MHz}, \mathrm{DMSO}-d_{6}\right): \delta 0.82\left(\mathrm{~s}, 3 \mathrm{H}, \mathrm{CH}_{3}\right), 1.03\left(\mathrm{~s}, 3 \mathrm{H}, \mathrm{CH}_{3}\right), 1.87-1.98(\mathrm{~m}, 4 \mathrm{H}$, $\left.2 \mathrm{CH}_{2}\right), 5.60(\mathrm{~s}, 1 \mathrm{H}, \mathrm{CH}), 9.75(\mathrm{~s}, 1 \mathrm{H}, \mathrm{NH}), 7.68-8.60(\mathrm{~m}, 4 \mathrm{H}, \mathrm{Ar}-\mathrm{H}), 10.82(\mathrm{~s}, 1 \mathrm{H}, \mathrm{NH}) .{ }^{13} \mathrm{C}$ NMR (400 MHz, DMSO-d $)_{6} \delta: 27.30\left(\mathrm{CH}_{3}\right), 29.15\left(\mathrm{CH}_{3}\right), 32.39(\mathrm{C}), 39.20\left(\mathrm{CH}_{2}\right), 50.28\left(\mathrm{CH}_{2}\right)$, $52.45(\mathrm{CH}), 103.57,116.39,124.37,128.22,128.75,129.52,129.87,131.20,131.38,146.32$ (Ar-C), $151.47(\mathrm{C}=\mathrm{N}), 175.47(\mathrm{C}=\mathrm{S}), 193.58(\mathrm{C}=\mathrm{O})$.

4-(tetrazolo[1,5-a]quinolin-4-yl)-2-thioxo-1,2,3,4,7,8-hexahydroquinazolin-5(6H)-one (5m). White solid, (73 \%), m.p. $>300{ }^{\circ} \mathrm{C}$, Anal. Calcd. for $\mathrm{C}_{17} \mathrm{H}_{14} \mathrm{~N}_{6} \mathrm{OS}$ : C 58.27, H 4.02, N 23.98\% Found C 58.18, H 4.15, N 24.12\%. IR (KBr, cm $\left.{ }^{-1}\right): 3377,3152$ (2NH), 2984 (ArC-H), 1687 $(\mathrm{C}=\mathrm{O}), 1618(\mathrm{C}=\mathrm{C}), 1643(\mathrm{C}=\mathrm{N}), 1184(\mathrm{C}=\mathrm{S}) .{ }^{1} \mathrm{H}$ NMR (400 MHz, DMSO-d $\left.)_{6}\right): \delta$ 1.78-2.12 (m, $\left.6 \mathrm{H}, 3 \mathrm{CH}_{2}\right), 5.67(\mathrm{~s}, 1 \mathrm{H}, \mathrm{CH}), 9.75(\mathrm{~s}, 1 \mathrm{H}, \mathrm{NH}), 7.69-8.58(\mathrm{~m}, 5 \mathrm{H}, \mathrm{Ar}-\mathrm{H}), 10.65(\mathrm{~s}, 1 \mathrm{H}, \mathrm{NH}) .{ }^{13} \mathrm{C}$ NMR (400 MHz, DMSO-d $)_{6} \delta: 21.15\left(\mathrm{CH}_{2}\right), 25.57\left(\mathrm{CH}_{2}\right), 35.71\left(\mathrm{CH}_{2}\right), 51.54(\mathrm{CH}), 104.72$, 116.59, 124.20, 127.92, 128.70, 129.76, 130.14, 131.09, 131.84, $146.59($ Ar-C), $151.48(\mathrm{C}=\mathrm{N})$, $175.68(\mathrm{C}=\mathrm{S}), 193.78(\mathrm{C}=\mathrm{O})$.

4-(7-methyltetrazolo[1,5-a]quinolin-4-yl)-2-thioxo-1,2,3,4,7,8-hexahydroquinazolin-5(6H)one (5n). White solid, (68 \%), m.p. $>300{ }^{\circ} \mathrm{C}$, Anal. Calcd. for $\mathrm{C}_{18} \mathrm{H}_{16} \mathrm{~N}_{6} \mathrm{OS}: \mathrm{C} 59.32, \mathrm{H} 4.42, \mathrm{~N}$ 23.06\% Found C 59.42, H 4.53, N 22.90\%. IR (KBr, cm $\left.{ }^{-1}\right): 3290,3210$ (2NH), 3008 (ArC-H), 
$2920(\mathrm{C}-\mathrm{H}), 1678(\mathrm{C}=\mathrm{O}), 1575(\mathrm{C}=\mathrm{C}), 1635(\mathrm{C}=\mathrm{N}), 1190(\mathrm{C}=\mathrm{S}) .{ }^{1} \mathrm{H}$ NMR $(400 \mathrm{MHz}$, DMSO$\left.d_{6}\right): \delta 1.85-2.20\left(\mathrm{~m}, 6 \mathrm{H}, 3 \mathrm{CH}_{2}\right), 3.37\left(\mathrm{~s}, 3 \mathrm{H}, \mathrm{CH}_{3}\right), 5.74(\mathrm{~s}, 1 \mathrm{H}, \mathrm{CH}), 9.73(\mathrm{~s}, 1 \mathrm{H}, \mathrm{NH}), 7.71-8.45$ (m, 4H, Ar-H), 10.75 (s, 1H, NH). ${ }^{13} \mathrm{C}$ NMR (400 MHz, DMSO-d 6 ) $\delta: 21.10\left(\mathrm{CH}_{3}\right), 21.42$ $\left(\mathrm{CH}_{2}\right), 26.59\left(\mathrm{CH}_{2}\right), 36.68\left(\mathrm{CH}_{2}\right), 51.35(\mathrm{CH}), 104.85,116.20,124.32,127.90,128.34,129.55$, 130.67, 132.70, 138.54, $146.39(\operatorname{Ar}-\mathrm{C}), 151.61(\mathrm{C}=\mathrm{N}), 175.49(\mathrm{C}=\mathrm{S}), 193.73(\mathrm{C}=\mathrm{O})$.

4-(7-methoxytetrazolo[1,5-a]quinolin-4-yl)-2-thioxo-1,2,3,4,7,8-hexahydroquinazolin-5(6H)one (5o). Off-white solid, (66 \%), m.p. $287-289^{\circ} \mathrm{C}$, Anal. Calcd. for $\mathrm{C}_{18} \mathrm{H}_{16} \mathrm{~N}_{6} \mathrm{O}_{2} \mathrm{~S}: \mathrm{C} 56.83, \mathrm{H}$ 4.23, N 22.09\% Found C 56.70, H 4.35, N 22.21\%. IR (KBr, cm $\left.{ }^{-1}\right): 3402,3181(2 \mathrm{NH}), 2940$ $(\operatorname{ArC}-\mathrm{H}), 1614(\mathrm{C}=\mathrm{C}), 1673(\mathrm{C}=\mathrm{O}), 1646(\mathrm{C}=\mathrm{N}), 1176(\mathrm{C}=\mathrm{S}), 1248 \& 1043\left(\mathrm{OCH}_{3}\right) .{ }^{1} \mathrm{H}$ NMR (400 MHz, DMSO-d $\left.)_{6}\right): \delta 1.80-2.28\left(\mathrm{~m}, 6 \mathrm{H}, 3 \mathrm{CH}_{2}\right), 3.95\left(\mathrm{~s}, 3 \mathrm{H}, \mathrm{OCH}_{3}\right), 5.72(\mathrm{~s}, 1 \mathrm{H}, \mathrm{CH}), 9.69$ $(\mathrm{s}, 1 \mathrm{H}, \mathrm{NH}), 7.75-8.65(\mathrm{~m}, 4 \mathrm{H}, \mathrm{Ar}-\mathrm{H}), 10.73(\mathrm{~s}, 1 \mathrm{H}, \mathrm{NH}) .{ }^{13} \mathrm{C}$ NMR $\left(400 \mathrm{MHz}\right.$, DMSO-d $\left.d_{6}\right) \delta$ : $21.14\left(\mathrm{CH}_{2}\right), 22.13\left(\mathrm{CH}_{2}\right), 28.22\left(\mathrm{CH}_{2}\right), 36.34(\mathrm{CH}), 55.71\left(\mathrm{OCH}_{3}\right), 104.87,116.54,124.45$, 127.80, 128.36, 129.52, 130.67, 132.65, 138.68, $146.44(\operatorname{Ar}-\mathrm{C}), 151.79(\mathrm{C}=\mathrm{N}), 175.69(\mathrm{C}=\mathrm{S})$, $193.56(\mathrm{C}=\mathrm{O})$.

4-(7-chlorotetrazolo[1,5-a]quinolin-4-yl)-2-thioxo-1,2,3,4,7,8-hexahydroquinazolin-5(6H)one (5p). Off-white solid, (60 \%), m.p. 294-296 ${ }^{\circ} \mathrm{C}$ Anal. Calcd. for $\mathrm{C}_{17} \mathrm{H}_{13} \mathrm{ClN}_{6} \mathrm{OS}$ : C 53.05, $\mathrm{H}$ 3.40, N 21.83\% Found C 53.21, H 3.29, N 21.92\%. IR (KBr, cm $\left.{ }^{-1}\right): 3320,3250(2 \mathrm{NH}), 2998$ $\left(\right.$ ArC-H), $1688(\mathrm{C}=\mathrm{O}), 1579(\mathrm{C}=\mathrm{C}), 1650(\mathrm{C}=\mathrm{N}), 755(\mathrm{C}-\mathrm{Cl}), 1178(\mathrm{C}=\mathrm{S}) .{ }^{1} \mathrm{H}$ NMR $(400 \mathrm{MHz}$, DMSO-d $\left.d_{6}\right): \delta 1.82-2.14\left(\mathrm{~m}, 6 \mathrm{H}, 3 \mathrm{CH}_{2}\right), 5.72(\mathrm{~s}, 1 \mathrm{H}, \mathrm{CH}), 9.75(\mathrm{~s}, 1 \mathrm{H}, \mathrm{NH}), 7.69-8.50(\mathrm{~m}, 4 \mathrm{H}$ $\mathrm{Ar}-\mathrm{H}), 10.82(\mathrm{~s}, 1 \mathrm{H}, \mathrm{NH}) .{ }^{13} \mathrm{C}$ NMR $\left(400 \mathrm{MHz}, \mathrm{DMSO}-d_{6}\right) \delta: 21.15\left(\mathrm{CH}_{2}\right), 26.45\left(\mathrm{CH}_{2}\right), 36.69$ $\left(\mathrm{CH}_{2}\right), 51.55(\mathrm{CH}), 104.98,116.59,124.26,128.26,128.82,129.87,130.20,130.78,131.96$, 146.86 (Ar-C), $151.68(\mathrm{C}=\mathrm{N}), 175.76(\mathrm{C}=\mathrm{S}), 193.52(\mathrm{C}=\mathrm{O})$.

\section{Acknowledgements}

The authors are thankful to the Department of chemistry, S P University for providing research facilities and Vaibhav laboratory, Ahmedabad for providing IR facilities.

\section{References}

1. Kappe, C. O. Eur. J. Med. Chem. 2000, 35, 1043.

2. Biginelli, P. Gazz. Chim. Ital. 1893, 23, 360.

3. Jauk, B.; Pernat, T.; Kappe, C. O. Molecules 2000, 5, 227.

4. Aron, Z. D.; Overman, L. E. Chem. Commun. 2004, 126, 253.

5. Jacqueline, C. B.; Patrica, A. M. J. Org. Chem. 2000, 65, 6777.

6. Li, M.; Guo, W. S.; Wen, L. R.; Li, Y. F.; Yang, H. Z. J. Mol. Catal. A: Chem. 2006, 258, 133. 
7. Choudhary, V. R.; Tillu, V. H.; Narkhede, V. S.; Borate, H. B.; Wakharkar, R. D. Catal. Commun. 2003, 4, 449.

8. Alexander Gross, G.; Wurziger, H.; Schober, A.; J. Comb. Chem. 2006, 8, 153.

9. Dondoni, A.; Massi, A.; Sabbatin, S.; Bertolasi, V. J. Org. Chem. 2002, 67, 6979.

10. Sabitha, G.; Reddy, G. S. K. K.; Reddy, C. S.; Yadav, J. S. Synlett 2003, 858.

11. Amini, M. M.; Shaabani, A.; Bazgir, A. Catal. Commun. 2006, 7, 843.

12. Rani, V. R.; Srinivas, N.; Kishan, M. R.; Kulkarni, S. J.; Raghavan, K. V. Green Chem. 2001, 3, 305 .

13. Bigi, F.; Carloni, S.; Frullanti, B.; Maggi, R.; Sartori, G. Tetrahedron Lett. 1999, 40, 3465.

14. Joseph, J. K.; Jain, S. L.; Sain, B.; J. Mol. Catal. A: Chem. 2006, 247, 99.

15. Sarac, S.; Yarim, M.; Ertan, M.; Kilic, F. S.; Erol, K. Pharmazie 2001, 56, 298.

16. Hassani, Z.; Islami, M. R.; Kalantari, M. Bio. \& Med. Chem. Lett. 2006, 16, 4479.

17. Kidwai, M.; Saxena, S.; Khalilur Rahman Khan, M.; Thukral, S. S. Eur. J. Med. Chem. 2005, $40,816$.

18. Yarim, M.; Sarac, S.; Kilic, F. S.; Erol, K. Il Farmaco 2003, 58, 17.

19. Yarim, M.; Sarac, S.; Ertan, M.; Kilic, F. S.; Erol, K. Arzneim. Forsch. 2002, 52, 27.

20. Kantevari, S.; Bantu, R.; Nagarapu, L. ARKIVOC 2006, (xvi), 136.

21. Patel, R. D.; Patel M. P.; Patel, R. G. Indian J. Chem. 2005, 44B, 1944.

22. Patel, N. A.; Surti, S. C.; Patel, R. G.; Patel, M. P. Phosphorus, Sulfur and Silicon 2008, 183, 2191.

23. Thakor, S.; Parmar, V. P.; Patel M. P.; Patel, R. G. Saudi Pharm. J. 2008, 16, 64.

24. Bekhit, A. A.; El-Sayed, O. A.; Aboulmagd, E.; Park, J. Y. Eur. J. Med. Chem.2004, 39, 249.

25. Dreikorn, B. A. U. S. Patent 3764 681, 1973.

26. Wright, T. L. U. S. Patent 4496 569, 1985.

27. Abdel-Gawad, S. M.; El-Gaby, M. S. A.; Ghorab, M. M. Il Farmaco 2000, 55, 287.

28. Abdel-Gawad, S. M.; El-Gaby, M. S. A.; Ghorab, M. M. Il Farmaco 2000, 55, 249.

29. Gupta, R.; Gupta, A. K.; Paul, S. Indian J. Chem. 2000, 39B, 847.

30. Srivastava, A; Singh, R. M. Indian J. Chem. 2005, 44B, 1868.

31. Collins, C. H.; Lyne, P. M. Microbial Methods, University Park Press: Baltimore, 1970.

32. Thangadurai, T. D.; Natarajan, K. Indian J. Chem. 2001, 40A, 573. 